\title{
Variações anatômicas na porção posterior do polígono de Willis
}

\author{
Anatomical variations in the posterior portion of the circle of Willis
}

\author{
Raiza Luna Peixotoa, Débora de Araujo Paza, João Luiz de Oliveira Gadelha Dantasa, \\ Maurus Marques de Almeida Holandab \\ a Graduandos de Medicina da Universidade Federal da Paraíba. \\ ${ }^{\text {b } M e ́ d i c o . ~ D o u t o r ~ e m ~ P r o d u t o s ~ N a t u r a i s ~ e ~ S i n t e ́ t i c o s ~ B i o a t i v o s ~ p e l a ~ U n i v e r s i d a d e ~ F e d e r a l ~ d a ~ P a r a i ́ b a . ~ P r o f e s s o r ~ d e ~ N e u r o l o g i a ~ d a ~ U n i v e r s i d a d e ~ F e d e r a l ~ d a ~ P a r a i ́ b a . ~}$
}

RESUMO Introdução: O encéfalo é vascularizado pelas artérias carótidas internas e vertebrais que, na base do crânio, formam um polígono anastomótico, o círculo arterial cerebral (CAC), também conhecido como polígono de Willis, fundamental para a irrigação cerebral. É frequente a ocorrência de variações anatômicas no CAC, muitas das quais se correlacionam ao surgimento de doenças cerebrovasculares.

Objetivos: O estudo objetivou identificar a prevalência das variações anatômicas das artérias da porção posterior do CAC com base em dados obtidos no exame necroscópico de cadáveres humanos do Serviço de Verificação de Óbitos localizado na Universidade Federal da Paraíba.

Materiais e Métodos: Foram dissecados 30 hemisférios cerebrais de cadáveres humanos de ambos os sexos, entre 18 e 70 anos. Posteriormente foram estudadas a circulação vértebro-basilar e a carotídea interna.

Resultados: Observou-se uma taxa de variação anatômica de $60 \%$ na circulação cerebral posterior. O hemisfério cerebral mais acometido por anormalidades foi o direito. As variações anatômicas foram mais prevalentes na artéria comunicante posterior e o tipo de variação anatômica mais freqüente foi a hipoplasia.

Conclusão: O conhecimento das peculiaridades das variações anatômica do CAC é fundamental, devido a sua grande prevalência. O seu estudo é imprescindível na compreensão do surgimento de doenças cerebrovasculares, como os aneurismas, na realização de procedimentos microvasculares reconstrutivos, bem como no fornecimento de informações essenciais nas avaliações radiológicas.

Palavras-chave: Círculo de Willis. Variação anatômica. Artéria basilar.

\section{ABSTRACT}

\begin{abstract}
Introduction: The brain is vascularized by the internal carotid and vertebral arteries, which form, at the base of the skull, an anastomotic polygon, the cerebral arterial circle (CAC), also known as the circle of Willis. The anatomical variation in the CAC is frequent, many of them being correlated to the occurrence of cerebrovascular diseases.

Objectives: This study aimed at identifying the prevalence of anatomical variations of the arteries of the posterior portion of the CAC based on data obtained from autopsies of cadavers of the Serviço de Verificação de Óbitos, located at Federal University of Paraiba.

Materials and Methods: 30 human cerebral hemispheres of both sexes between 18 and 70 years of age were dissected. Next, the vertebrobasilar and the internal carotid circulation were studied.

Results: There was a rate of $60 \%$ of abnormalities in the anatomic posterior cerebral circulation. The cerebral hemisphere more affected was the right one. Anatomical variations were more prevalent in the posterior communicating artery, and the type of anatomical variation that occurred most frequently was hypoplasia.

Conclusion: Knowing the peculiarities of the anatomical variations of CAC is fundamental, due to its high prevalence and its essentiality to understand the emergence of cerebrovascular diseases, such as aneurysms, to perform microvascular reconstructive procedures, as well as to provide essential information on radiological examinations.

Keywords: Circle of Willis. Anatomic variation. Basilar artery.
\end{abstract}




\section{INTRODUÇÃO}

O sistema nervoso central ( $\mathrm{SNC}$ ) é constituído por estruturas biológicas nobres, altamente especializadas e de papel central no organismo humano. Embora represente aproximadamente $2 \%$ do peso corporal total, sua elevada taxa metabólica requer um intenso e permanente suprimento hemodinâmico para prover o chamado acoplamento metabólico cerebral ${ }^{1}$.

O círculo arterial cerebral (CAC), também conhecido como o círculo de Willis, foi descrito pelo anatomista e fisiologista inglês Thomas Willis em 1664, como uma anastomose arterial de forma poligonal situado na base do cérebro, onde circunda o quiasma óptico, o túber cinéreo e relaciona-se ainda com a fossa interpenduncular e a substância perfurada anterior. Este é formado pelas porções proximais das artérias cerebrais anterior, média e posterior, pela artéria comunicante anterior (ACOA) e pelas artérias comunicantes posteriores (ACoP) direita e esquerda. A artéria comunicante anterior é pequena e anastomosa as duas artérias cerebrais anteriores adiante do quiasma óptico. As artérias comunicantes posteriores unem de cada lado as carótidas internas com as cerebrais posteriores correspondentes.

Deste modo elas anastomosam o sistema carotídeo interno ao sistema vértebrobasilar. Entretanto esta anastomose é apenas potencial, pois, em condições normais, não há passagem significativa de sangue do sistema vertebral para o carotídeo interno ou vice-versa. Do mesmo modo, praticamente, não existe troca de sangue entre as metades direita e esquerda do círculo arterial ${ }^{1}$.

O fluxo sanguíneo das artérias cerebrais posteriores (ACP) provém primariamente da artéria basilar (em 70\% dos casos) e da artéria carótida interna ( $10 \%$ dos casos), nos $20 \%$ restantes uma ACP é irrigada pela artéria carótida interna e a outra pela artéria basilar. A ACP passa dorsalmente ao terceiro par craniano, atravessa os pedúnculos cerebrais e, a seguir, ascende ao longo da borda medial do tentório, onde se ramifica em divisão anterior e posterior. A divisão anterior irriga a superfície inferior do lobo temporal, onde os seus ramos terminam fazendo anastomose com a artéria cerebral média (ACM). A divisão posterior irriga o lobo occipital, onde seus ramos terminais fazem anastomose com a ACA e ACM.

A função do polígono de Willis é manter um fluxo sanguíneo adequado em caso de obstrução. Na maioria dos casos há algumas variações dessa definição anatômica original. Em uma grande série de autópsias de indivíduos normais, mais da metade apresentou círculo de Willis incompleto ${ }^{2}$. O local mais comum dessas anormalidades, que amiúde se manifestam na forma de hipoplasia e atresia, consiste nas artérias comunicantes posteriores $(20 \%)$ e nas cerebrais anteriores $(10 \%)^{2}$. Estudos prévios mostram que pacientes que possuem variantes do círculo com circulação colateral eficiente tem um menor risco de ataque isquêmico transitório e acidente vascular cerebral do que pacientes sem estas colaterais ${ }^{3-4}$. Alguns estudos propuseram a correlação entre variantes do CAC e algumas doenças cerebrovasculares e também diferenças na incidência dessas doenças em populações diferentes. Dessa forma, cogita-se que a distribuição diferente de variações do CAC pode explicar parcialmente a incidência distinta de algumas doenças cerebrovasculares em grupos étnicos ou raciais diferentes ${ }^{5}$.

Outros estudos encontraram uma correlação entre aneurisma cerebral e certas alterações anatômicas vasculares e estas, provavelmente, estão implicadas na patogênese de aneurismas intracranianos congênitos, podendo atuar como time markers de possíveis eventos negativos durante a vasculogênese ${ }^{6-7}$. A relevância da pesquisa se mostra evidente, pois o conhecimento minucioso da circulação carotídea e vértebrobasilar podem esclarecer, em parte, tais questionamentos ainda presentes na literatura científica.

Objetivou-se, portanto, identificar a prevalência de variações anatômicas das artérias da porção posterior do circulo de Willis com base em dados obtidos no exame necroscópico de cadáveres humanos do Serviço de Verificação de Óbitos do Estado da Paraíba (SVO-PB), anexo à Universidade Federal da Paraíba (UFPB).

\section{MATERIAIS E MÉTODOS}

Foi conduzido um estudo do tipo série de casos, baseado no estudo anatômico da circulação arterial cerebral de cérebros submetidos à necropsia no Serviço de Verificação de Óbito (SVO-PB) localizado na UFPB. Foram incluídos na pesquisa indivíduos entre 18 e 70 anos. Como critérios de exclusão, tivemos indivíduos com história de trauma de crânio, ou que tiveram antecedentes de cirurgias neurológicas, como também, presença de doenças que alteram a anatomia e dificultam a observação das artérias.

O processo de amostragem foi do tipo não probabilístico por conveniência. Este processo de seleção de unidades amostrais tem a vantagem de ser mais facilmente acessível, rápido e menos dispendioso. A amostra total do estudo foi composta por 15 cérebros de cadáveres humanos (totalizando 30 hemisférios cerebrais). As artérias do círculo de Willis foram retiradas no momento da necropsia, sendo removidas a partir da emergência das artérias vertebrais no crânio através do forame magno. Os cérebros eram sempre dissecados pelo mesmo grupo de pessoas, bem como a avaliação das variações anatômicas. 
Nesse estudo foram avaliadas as seguintes variações anatômicas da circulação arterial cerebral posterior: (1) PICA extradural: a PICA tem origem angiográfica no segmento extradural da artéria vertebral; (2) Assimetria do topo da basilar: quando os segmentos $\mathrm{P} 1$ da circulação posterior têm alturas diferentes na projeção angiográfica frontal; (3) Fenestração: quando um segmento arterial apresenta dois canais distintos, próximos e paralelos, os quais possuem, histologicamente, uma única túnica externa. Em relação à artéria basilar, o termo mais apropriado seria fusão incompleta, já que esse vaso tem a peculiaridade de originarse da fusão de duas artérias longitudinais embrionárias; (4) Duplicação: quando dois canais vasculares, não necessariamente próximos e paralelos, cursando envoltos por túnicas distintas, tocam-se proximal e distalmente; (5) Hiplopasia: quando um segmento arterial apresenta calibre inferior a 50\% do correspondente contralateral; (6) Dilatação juncional de ACoP: também conhecida como dilatação infundibular. São alargamentos que afetam a origem da AcoP, em sua junção com a artéria carótida interna; (7) ACoP fetal: quando a artéria comunicante posterior permanece com o padrão observado na circulação fetal, de calibre maior que o normal.

O projeto foi registrado em Comitê de Ética em Pesquisa, observando-se todas as diretrizes e normas regulamentadoras para pesquisa envolvendo seres humanos, estabelecidas na Resolução no 196/96 do Conselho Nacional de Saúde, em vigor no país, principalmente no que diz respeito ao consentimento livre e esclarecido do participante, bem como da garantia do seu anonimato e o sigilo de dados confidenciais, que foi assinado pelo familiar, já que se tratou de um estudo com cadáveres (protocolo no 150/10 do Comitê de Ética em Pesquisa - HULW em 05.04.2010).

Os dados foram armazenados em planilhas do programa Microsoft Excel 2007 inicialmente e ao término da pesquisa foram analisados pelo Programa Estatístico SPSS (Statistical Package for Social Sciente), versão 15.0. Para análise dos dados utilizamos apenas a análise descritiva. Foram elaboradas tabelas de frequência para as variáveis categóricas (sexo e idade, por exemplo) com valores de frequência absoluta (N) e percentual (\%).

\section{RESULTADOS}

A casuística foi composta de 30 hemisférios cerebrais, dissecados por ocasião da necropsia, sendo 18 (60\%) do sexo masculino e $12(40 \%)$ do sexo feminino. A média de idade foi de 54,12 anos.

No estudo, foi possível identificar 18 variações anatômicas entre os 30 hemisféricos cerebrais analisados. A taxa de diferenciação entre o observado e a descrição anatômica original foi de $60 \%$. Houve discrepância no índice de acometimento entre os hemisférios. Enquanto o hemisfério direito contou com 13 variações, o esquerdo apresentou apenas 6 delas.

O tipo de variação anatômica mais prevalente foi a hipoplasia da AcoP (55,5\%), seguida da AcoP fetal (16,6\%). Não foram evidenciadas variações anatômicas do tipo: assimetria do topo a basilar, fenestração, PICA extradural, dilatações juncionais, duplicação e agenesia da AcoP. Foram encontradas alterações não esperadas: um caso de AICAPICA e outra variação ao nível de bifurcação da artéria cerebral posterior (Figura 1).

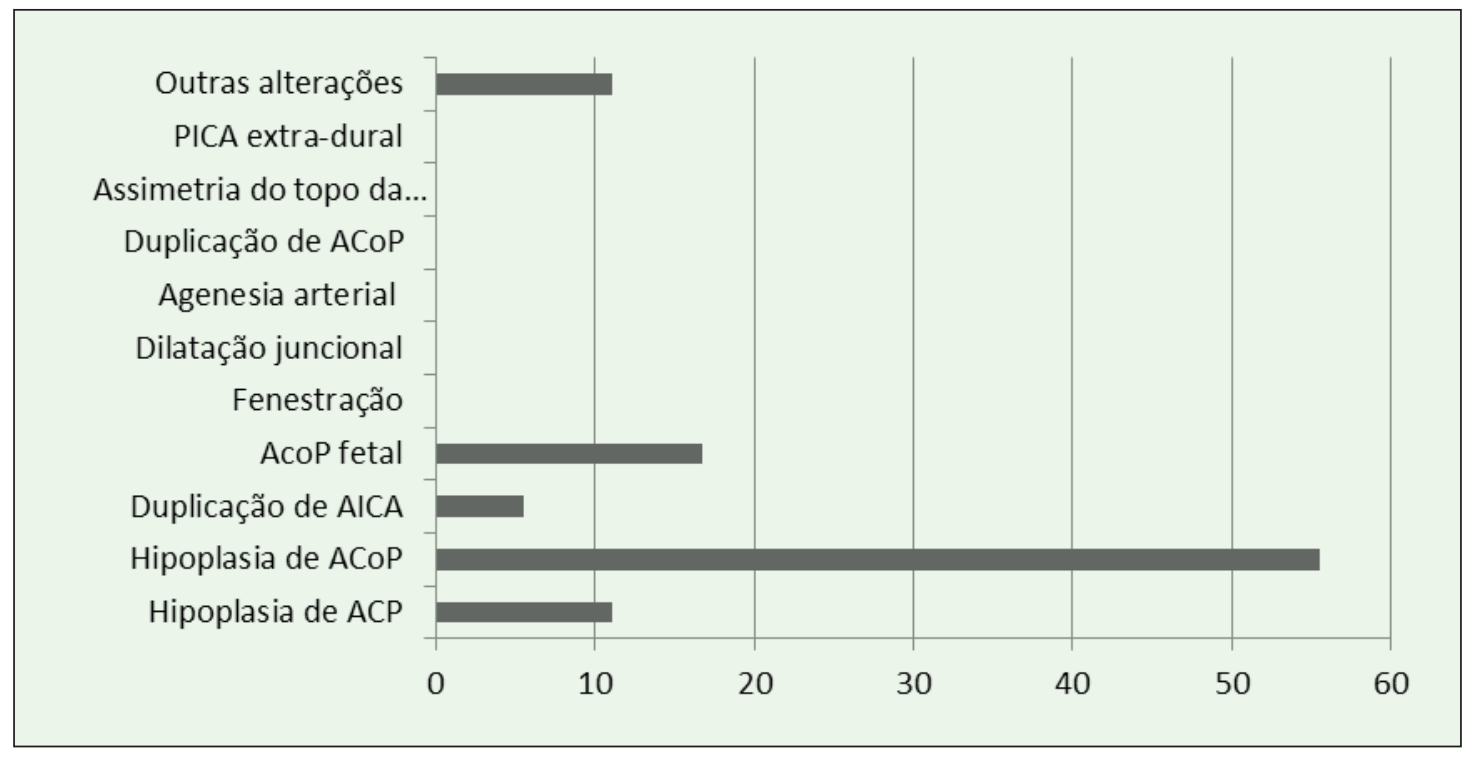

Figura 1. Prevalência das variações anatômicas no círculo arterial cerebral (\%). 


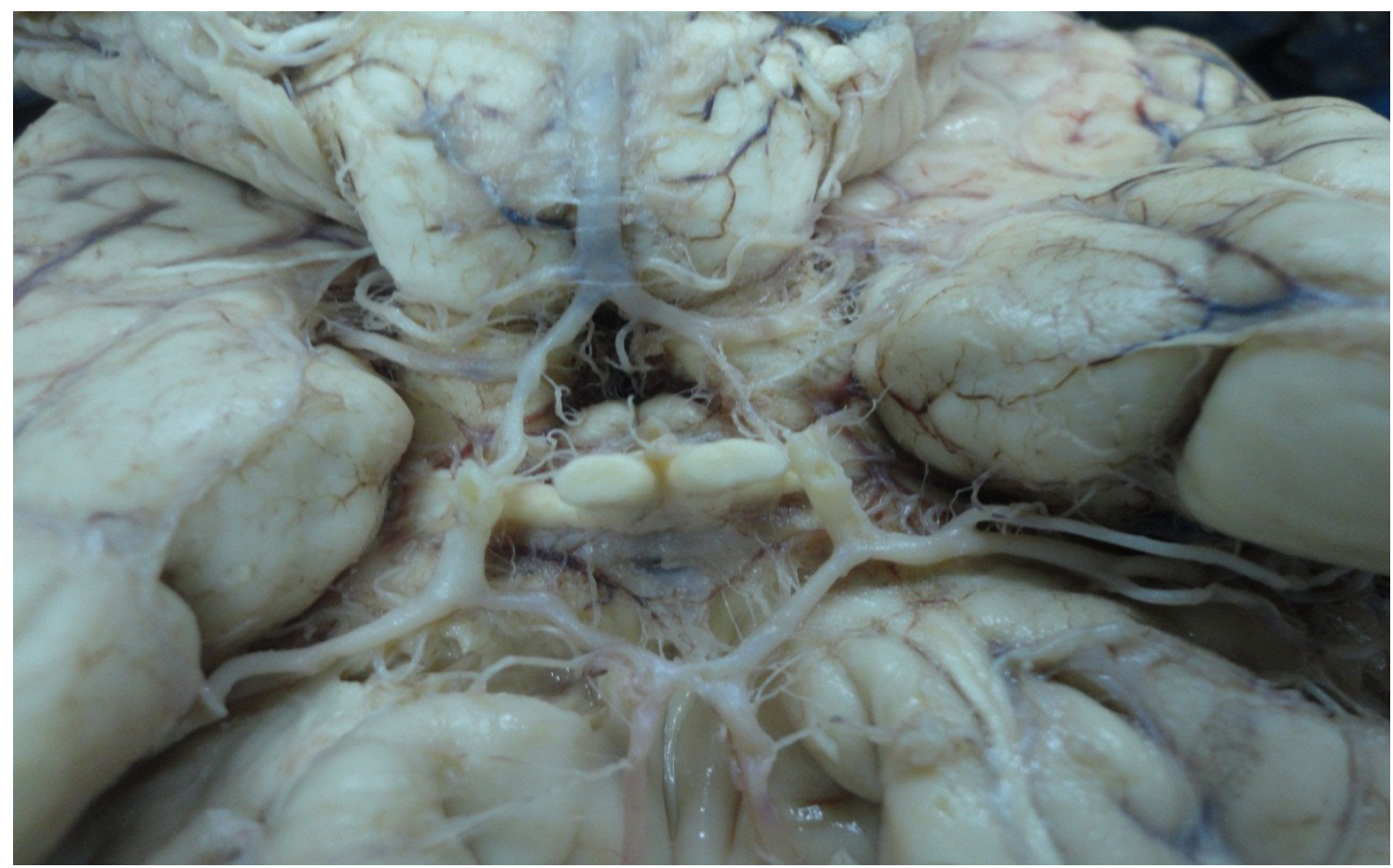

Figura 2. Vista ântero-posterior do círculo arterial cerebral. Nota-se hipoplasia de artérias comunicantes posteriores (seta).

O segmento vascular do território posterior mais acometido foi a artéria comunicante posterior, local onde foram observadas $72,2 \%$ das alterações. Não foram observadas alterações: na artéria basilar (AB), nas vertebrais (AV), nem na artéria cerebelar póstero-inferior (PICA). A variação mais prevalente encontrada no estudo está ilustrada na Figura 2.

\section{DISCUSSÃO}

O presente estudo buscou avaliar a presença de variações anatômicas na circulação posterior do polígono de Willis e identificou a presença de 18 anomalias em 30 hemisférios cerebrais.

Outros dados da literatura corroboram com este achado. Segundo Krishnamurthy et al. ${ }^{8}$, as variações no polígono de Willis são muito comuns, sendo a sua descrição clássica encontrada muito raramente. Cecil ${ }^{2}$ refere ser muito frequente o achado de variações anatômicas no círculo arterial cerebral. Soares et al. ${ }^{9}$, em seu estudo anatômico e morfométrico dos vasos que constituem o polígono de Willis, identificou a presença de onze anormalidades, em todo o polígono, em nove encéfalos.

No presente estudo as alterações mostraram-se mais presentes no hemisfério cerebral direito, onde foram observados onze das dezoito alterações arteriais. Não foram encontrados dados na literatura acerca desse parâmetro, sugerindo uma maior necessidade de investigação a esse respeito. O segmento vascular mais acometido foi a artéria comunicante posterior (ACoP), seguido pela artéria cerebral posterior e artéria cerebelar anterior inferior. Cecil ${ }^{2}$ preconiza que os locais mais comuns de anormalidades seriam as artérias comunicantes posteriores (22\%) e artérias cerebrais anteriores (10\%). Eftekhar et al. ${ }^{5}$, em seu trabalho, também aponta a artéria comunicante posterior como o local mais comum de anormalidades.

Em estudo publicado por Bisaria ${ }^{10}$ em 1984, com 126 cadáveres, foi concluído que a variação mais comum da artéria comunicante posterior seria a AcoP fetal, sendo descrita em $31.7 \%$ dos casos. Ainda, a dilatação juncional foi considerada um achado importante, já que se fez presente em 6,3\% dos casos. Já os estudos de Shomer et al. ${ }^{11}$, em 1994, mostram elevada prevalência de hipoplasia de AcoP. Esse achado é de grande repercussão clínica, já que o menor diâmetro da AcoP está relacionado ao maior risco de acidente vascular isquêmico.

Şengül et al. ${ }^{12}$ descreveram que a AcoP estaria normal em $66,6 \%$ dos casos; em 26,6\% ela estaria fetal (hiperplásica) e em e em 6,7\% ela estaria hipoplásica. Em seu estudo, não foram encontrados casos de ausência ou de duplicação da AcoP. De Silva ${ }^{13}$ também encontrou alta prevalência de hipoplasia de AcoP em seu estudo no Sri Lanka. Merkkola ${ }^{14}$ notificou a ausência da AcoP em $46 \%$ dos casos, enquanto o nosso estudo não conseguiu evidenciar nenhuma agenesia dessa artéria em nossa casuística. 
Kapoor et al. ${ }^{16}$ avaliaram 1000 espécimes, sendo que 452 cérebros $(45,2 \%)$ apresentaram um círculo de Willis típico e em $54,8 \%$ observaram-se variações. Alterações hipoplásicas da artéria cerebral posterior foram encontradas em 10,6\% dos casos, enquanto a sua multiplicidade foi relatada em apenas $2,4 \%$. Os achados para os outros vasos foi o seguinte: a artéria cerebral anterior estava ausente em $0,4 \%$, era hipoplásica em 1,7\%, era duplicada em 2,6\%, triplicada em 2,3\% e única em 0,9\%; a artéria comunicante anterior estava ausente em $1,8 \%$, duplicada em $10 \%$ e triplicada em 1,2\%; a artéria comunicante posterior estava ausente em $1 \%$ e hipoplásica em 13,2\% casos.

A vascularização do sistema nervoso central tem sido estudada há anos por diversas linhas de pesquisas em neurociências. O conhecimento detalhado dessa complexa trama vascular é de fundamental importância para a compreensão de diversas síndromes clínicas em neurologia, planejamento e execução segura de intervenções endovasculares e cirurgias abertas, além de especulação de prognósticos frente ao dano vascular agudo. Anomalias no círculo de Willis parecem ter um papel importante no desenvolvimento de aneurismas, produzindo alterações hemodinâmicas no fluxo sanguíneo cerebral e induzindo pressão sobre o ponto fraco das artérias em zonas de bifurcação. Além disso, o conhecimento dessas variações permite evitar iatrogenias neurocirúrgicas.

No que concerne à circulação posterior, a artéria comunicante posterior parece ser o local de maior índice de variações. A hipoplasia foi a alteração mais documentada em nosso estudo.

No nosso trabalho, encontramos uma prevalência de variações anatômicas de $60 \%$, sendo a mais frequente delas a hipoplasia da AcoP. Devido ao baixo número de encéfalos dissecados e amostragem aleatória, não podemos fazer generalização externa. Assim mais estudos anatômicos de base populacional necessitam ser realizados para definir a real prevalência de cada variação anatômica, bem como estudar a relação delas com doenças cerebrovasculares.

\section{REFERÊNCIAS}

1. Machado A. Neuroanatomia funcional. $2 \underline{a}$ ed. São Paulo: Atheneu; 2006.

2. Cecil L. Textbook of Medicine. 20th ed. Philadelphia: W. B Company; 2005

3. Henderson RD, Eliasziw M, Fox AJ, Rothwell PM, Barnett HJ. Angiographically defined collateral circulation and risk of stroke in patients with severe carotid artery stenosis. Stroke. 2000;31:128-32. http://dx.doi.org/10.1161/01.STR.31.1.128
4. Horikoshi T, Akiyama I, Yamagata Z, Sugita M, Nukui H. Magnetic resonance angiographic evidence of sex-linked variations in the circle of willis and the occurrence of cerebral aneurysms J Neurosurg. 2002;96(4):697-703. http://dx.doi.org/10.3171/ jns.2002.96.4.0697

5. Eftekhar B, Dadmehr M, Ansari S, Mohammad Ghodsi, Bashir Nazparvar, Ebrahim Ketabchi . Are the distributions of variations of circle of Willis different in different populations? - Results of an anatomical study and review of literature. BMC Neurology. 2006; 6:1-9. http://dx.doi.org/10.1186/1471-2377-6-22

6. Miyazaki S, Kamata K, Yamaura A. Multiple aneurysms of the vertebrobasilar system associated with fenestration of the vertebral artery. Surg Neurol. 1981;15(3):192-5. http://dx.doi. org/10.1016/0090-3019(81)90140-3

7. Mazighi M, Porter PJ, Rodesch G, Alvarez H, Aghakhani N, Lausiaunias P. Vascular anomalies and the risk of multiple aneurysms development and bleeding. Interv Neuroradiol. 2002;8(1):15-20.

8. Krishnamurthy A, Rao CP, Narayana K, Nayak SR, Kumar SM, Surendran S. Circulus arteriosus cerebri: a study of variation in the fetal and adult human brains of south Indians. Morphologie. 2006;90(290): 139-43. http://dx.doi.org/10.1016/S1286-0115(06)74494-0

9. Soares JC, Tamega OJ, Schwerdtfeger W, Cintra MD, Cury PR. Estudo anatômico e morfométrico dos vasos que constituem o Polígono de Willis. Rev Bras Ciênc Morfol. 1994;11(2):115-24

10. Bisaria KK. Anomalies of the posterior communicating artery and their potential clinical significance. J Neurosurg. 1984;60(3):572-6. http://dx.doi.org/10.3171/jns.1984.60.3.0572

11. Shomer DF, Marks MP, Steinberg G, Johnstone IM, Boothroyd DB, Ross MR, Pelc NJ, Enzmann DR. The anatomy of the posterior communicating artery as a risk factor for ischemic cerebral infarction. N Engl J Med.1994;330(22):1565-70. http://dx.doi.org/10.1056/ NEJM199406023302204

12. Şengül G, Akar A, Aydin IH. Intraoperative Observations of Anatomic Variations of the Posterior Communicating Artery: a microsurgical approach. J Neurol Sci. 2009;26:279-85

13. De Silva KR, Silva R, Amaratunga D, Gunasekera WS, Jayesekera RW. Types of the cerebral arterial circle (circle of Willis) in a Sri Lankan population. BMC Neurol. 2011;11:5. http://dx.doi. org/10.1186/1471-2377-11-5

14. Merkkola P, Tulla H, Ronkainen A, Soppi V, Oksala A, Koivisto $\mathrm{T}$, Hippelainen $\mathrm{M}$. Incomplete circle of Willis and right axillary artery perfusion. Ann Thorac Surg. 2006;82:74-9. http://dx.doi. org/10.1016/j.athoracsur.2006.02.034

15. Caruso G, Vincentelli F, Rabehanta P, Giudicelli G, Grisoli F. Anomalies of the P1 segment of the posterior cerebral artery: early bifurcation or duplication, fenestration, common trunk with the superior cerebellar artery. Acta Neurochir (Wien). 1991;109: 66-71. http://dx.doi.org/10.1007/BF01405701

16. Kapoor K, Singh B, Dewan LI. Variations in the configuration of the circle of Willis. Anat Sci Int. 2008;83:96-106. http://dx.doi. org/10.1111/j.1447-073X.2007.00216.x 